\title{
The Eeklo Modified Subvastus Approach for Total Knee Arthroplasty: Detailed Surgical Tips and Tricks
}

\author{
Ignace Ghijselings, $M D^{1}$, Hans Van den Wyngaert, $M D^{1}$, Alex Demurie Alex, $M D^{1}$, Stefaan Van Onsem, \\ $M D, P h D^{1}$ and Hendrik P. Delport, MD, PhD ${ }^{*}$
}

${ }^{1}$ Department of Orthopaedics and Traumatology, AZ Alma, Eeklo, Belgium

${ }^{2}$ Division of Orthopedics, University Hospitals Leuven, Belgium

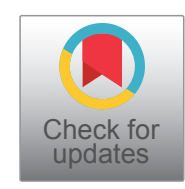

\begin{abstract}
This paper describes step by step a modified Far Medial Subvastus approach-the Eeklo Modified Subvastus approach (EMS)-for Total Knee Arthroplasty (TKA). The EMS approach is a modification of the classic Subvastus approach. It provides excellent exposure of the knee joint with preservation of all peri-articular soft tissues.

Avoiding transection of the infrapatellar nerve branches is a significant factor in patient satisfaction. The blood supply of the patella is preserved resulting in less patellar necrosis and fracture or loosening of the patellar component. The extensor mechanism remains completely intact which maintains quadriceps strength and function. Painful scar tissue can form post-surgery at the level of the skin or deeper. The EMS incision and arthrotomy avoids all bony prominences preventing limitation of flexion in case of induration of healing cicatrices.

This combination of advantages ensures rapid recovery, better knee range of motion and finally excellent patient satisfaction. With closure of the EMS approach the periarticular soft tissue strains are restored to those typical of the native knee while intra-articular bleeding stops quickly due to complete sealing of the joint.

Indications of the EMS approach are primary TKA and revision surgery in selected patients. The EMS approach is an improved modification of the Far Medial Subvastus Approach and can become the New Golden Standard for TKA.

Level of evidence $V$

Keywords

Subvastus, Surgical approach, Total knee arthroplasty, Surgical tips

\section{List of Abbreviations}

EMS: Eeklo Modified Subvastus Approach; TKA: Total Knee Arthroplasty; MCL: Medial Collateral Ligament; LCL: Lateral Collateral Ligament; VMO: Vastus Medialis Obliquus; MPFQ-complex: Medial Patello Femoral Quadriceps Complex; DGA: Descending Genicular Artery; MPTL: Medial Patellar Tibial Ligament; IPFP: Infrapatellar Fat Pad; ACL: Anterior Cruciate Ligament; PCL: Posterior Cruciate Ligament; IPP: Infrapatellar Plica; PIPB: Patella-In-Place Balancer; MIS: Minimal Invasive Surgery
\end{abstract}

\section{Introduction}

Despite the fact that many designs of TKA are today available on the market, none of these seems to be associated with a patient satisfaction rate higher than $80 \%$. Recent improvements in surgical technique in order to improve the accuracy in alignment using better instruments and navigation has decreased the proportion of outliers [1-3]. Improved ligament balancing techniques with all kinds of tensioners without the patella in place have not been able to provide reproducible results in the hands of most orthopedic surgeons [4]. We believe that the preservation of the periarticular soft tissues as well as the patella in place during balancing plays a very important role.
The concept of isometry of the collateral ligaments is decisive. It has been shown by J Victor, et al. that the Medial

*Corresponding author: Prof. Dr. Hendrik Delport P, MD, PhD, Division of Orthopedics, University Hospitals Leuven, August de Boeckstraat 1/8, B-9100 Sint-Niklaas, Belgium

Accepted: March 20, 2021

Published online: March 22, 2021

Citation: Ghijselings I, Van den Wyngaert H, Demurie A, et al. (2021) The Eeklo Modified Subvastus Approach for Total Knee Arthroplasty: Detailed Surgical Tips and Tricks. J Orthop Surg Tech 4(1):246-253

Copyright: (c) 2021 Ghijselings I, et al. This is an open-access article distributed under the terms of the Creative Commons Attribution License, which permits unrestricted use, distribution, and reproduction in any medium, provided the original author and source are credited. 
Citation: Ghijselings I, Van den Wyngaert H, Demurie A, et al. (2021) The Eeklo Modified Subvastus Approach for Total Knee Arthroplasty: Detailed Surgical Tips and Tricks. J Orthop Surg Tech 4(1):246-253

Collateral Ligament (MCL) and the Lateral Collateral Ligament (LCL) in the intact knee joint remain isometric during several motor tasks [5]. In a cadaver study, we have investigated the specific soft tissue envelope before and after knee joint replacement.

Ideally one would assume that after TKA the periarticular soft tissue strains could be restored to those typical of the native knee $[6,7]$.

It has been demonstrated by Zimny that soft tissue strain is correlated with neuroreceptor activation and proprioception [8].

In view of this, the clinical implication of the results obtained in our study suggested that correction to constitutional rather than neutral alignment is preferable, since the soft tissue strain patterns are better restored when constitutional alignment is obtained.

Surgical techniques, even after the learning curve, show a continuous evolution. Orthopedic surgeons strive to improve the technical aspects of their interventions to ameliorate clinical outcome and patient satisfaction. Both senior authors each having a vast experience of $6000+$ TKA procedures using a subvastus approach experienced a gradual evolution of their surgical details. Most credit however deserves the first author who developed many small details of this approach in his orthopedic practice.

We are convinced that it is wise to avoid ligament release procedures in balancing the flexion/extension gap. If we need to perform a ligament release, we are convinced that we made some error in the positioning of the components. Ligament releases era in TKA should be over now.

A good way to start is using a surgical approach that preserves the soft tissue envelope as much as possible.

\section{New Surgical Technique}

Nowadays, the authors performa Subvastus Surgical Technique as developed by the first author also known as the "Eeklo Modified Sub-vastus" (EMS).

We propose General Anesthesia for maximal muscle relaxation and control of blood pressure. The patient is positioned supine in the standard fashion. No tourniquet is applied. We employ a post to support the foot, such that the knee can be flexed and maintained in full flexion.

The skin incision for the EMS is made one $\mathrm{cm}$. medial to the medial border of the patella and the medial side of the tibial tubercle with the knee in deep flexion (Figure 1).

The length of the incision is not minimal and should have the intention to circumvent all traction on the skin corners. In the medio-lateral plan it runs $1-1.5 \mathrm{~cm}$. medial of the medial border of the patella. This area constitutes of under laying more compressible tissue averting bony prominences in view of the later scar formation. It starts approximately $10 \mathrm{~cm}$. proximal of the superior pole of the patella, extending to the joint line.

The incision is carried sharply through the subcutane-

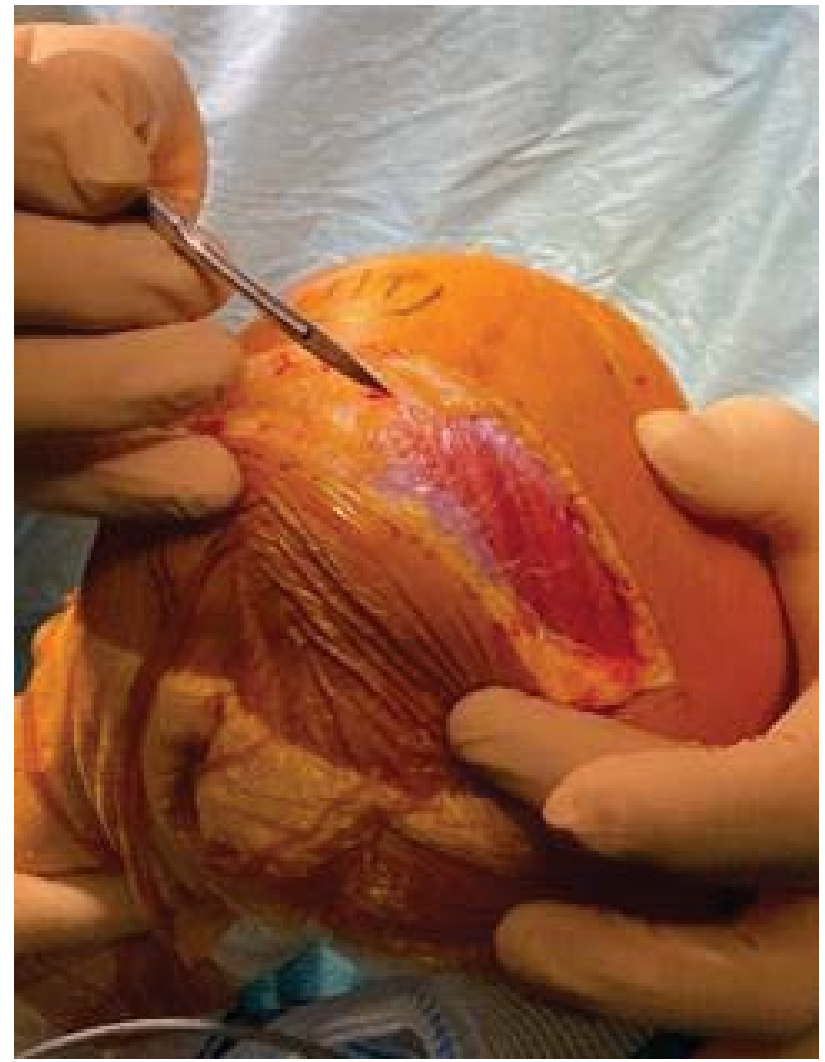

Figure 1: Skin incision.

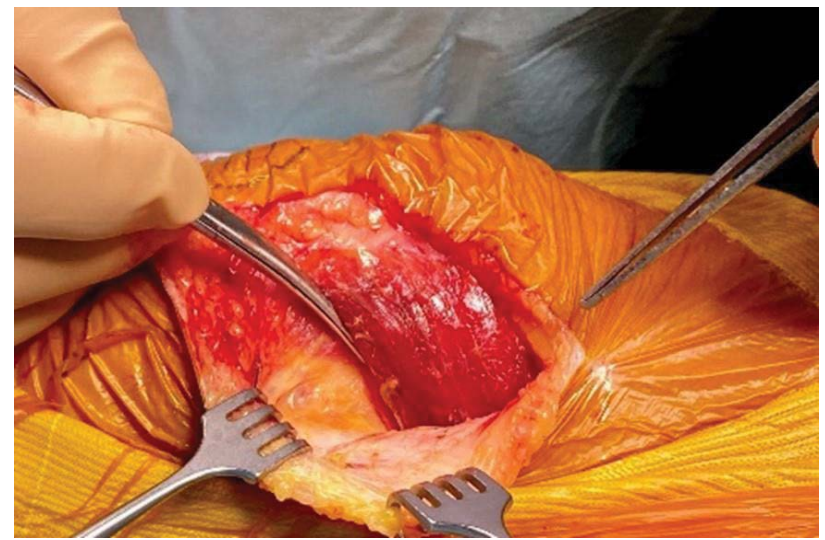

Figure 2: Releasing the VMO.

ous tissue down to the muscle fascia of the Vastus Medialis Obliquus (VMO). Full-thickness medial and lateral flaps containing skin, subcutaneous fat and fascia are then created sharply. No coagulation is done in this layer also to prevent skin necrosis.

Next, the distal insertion of the VMO on the patella is exposed. The fascia overlying the VMO is released sharply, taking care not to injure any underlying muscle fibers, simultaneously careful hemostasis of the perforating arteries entering the muscle is indicated. To allow mobilization the VMO is released from the medial intermuscular septum in the proximal direction (Figure 2). 


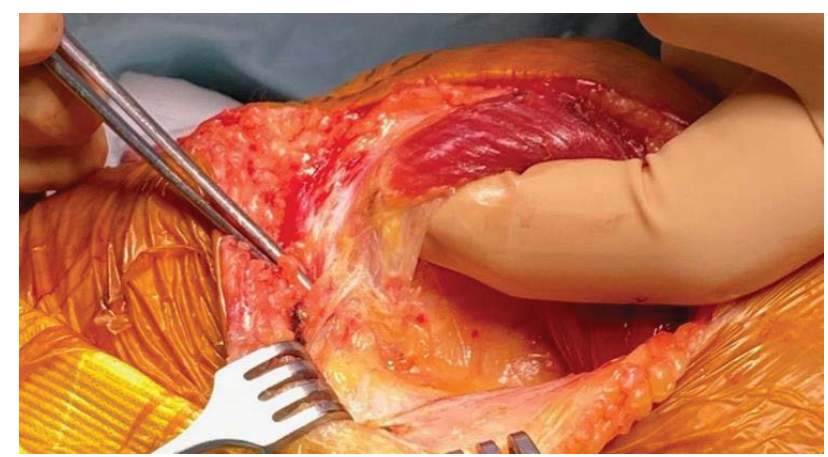

Figure 3: Identification of the Medial Patello Femoral Quadriceps Complex.

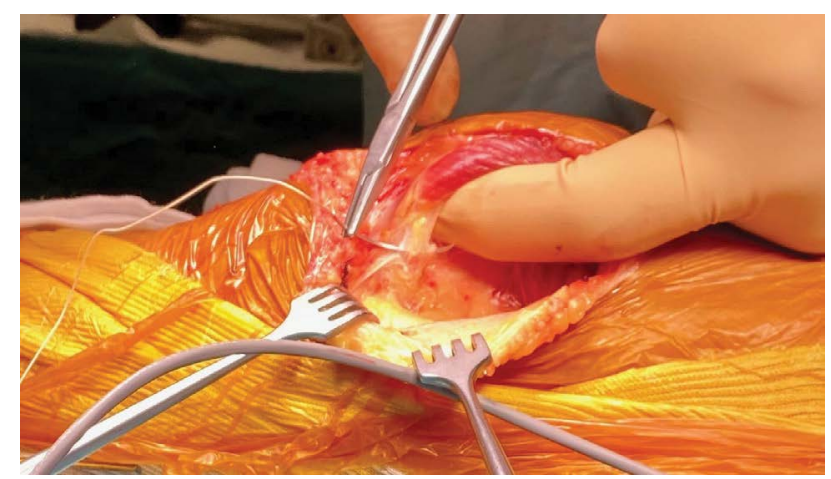

Figure 4: Placing the reference sutures.

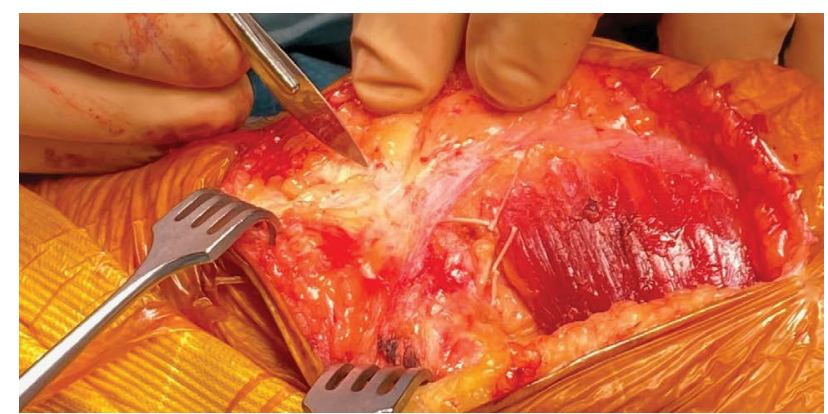

Figure 5: Finding the distal border of the MPFQ-complex.

Using blunt dissection, a finger can be placed underneath the VMO at its inferior border. The VMO is then lifted while maintaining its attachment through the Medial Patello Femoral Quadriceps Complex (MPFQ-complex) (Figure 3). The MPFQ-complex is a crucial soft tissue element that needs to be repaired. It contains in addition a medial patellotibial ligament and a medial patellomeniscal ligament [9-12].

Two sutures are placed to serve as a reference to reattach the MPFQ-complex at the end of the surgical intervention and to ligate the Descending Genicular Artery (DGA) (Figure 4).

Following step is to incise the medial retinaculum at the medial border of the patellar tendon. A fat spot appears at the distal border of the MPFQ-complex (Figure 5).

Incise now the MPFQ between the two ligation points but

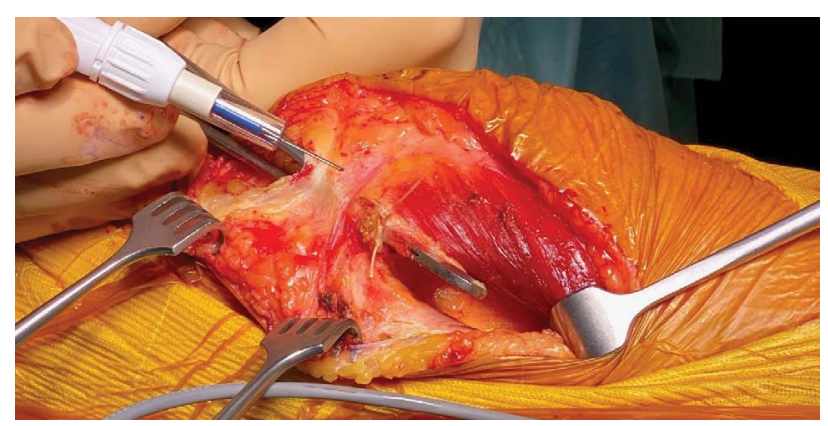

Figure 6: Separate the MPFQ.

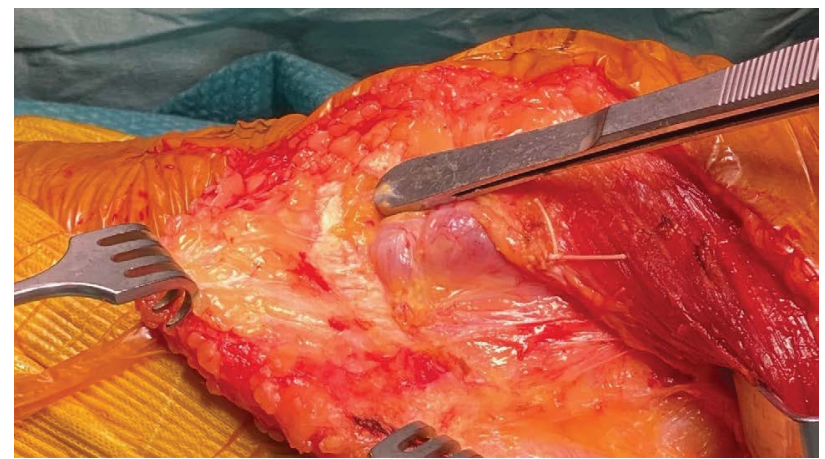

Figure 7: Preserve the infrapatellar nerve.

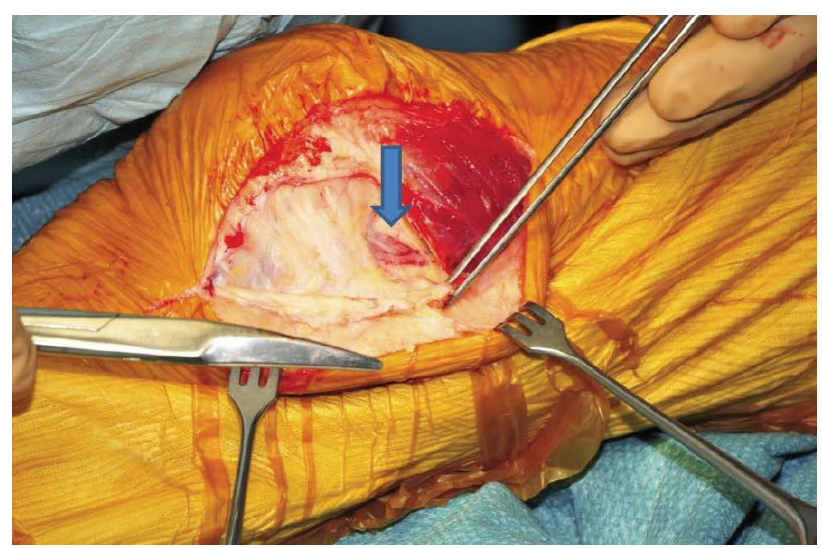

Figure 8: Identify "the 3 friends".

remain extra-synovial. One can use tweezers to separate the layers (Figure 6).

Insert now a protector distally (under the retinaculum but above the mucosa), until resistance is sensed. This stop is the attachment of the Pes Anserinus. A blunt top down forceps can be used to dissect the anatomical plane between the retinaculum and the underlying synovium, the retinaculum only is sharply divided with a knife from inside to outside taking care not to damage the subcutaneous fat containing the infrapatellar branch of the saphenous nerve (Figure 7).

The medial retinaculum is now separated from the underlying medial synovial tissue by blunt dissection. The synovium remains intact (Figure 8). 


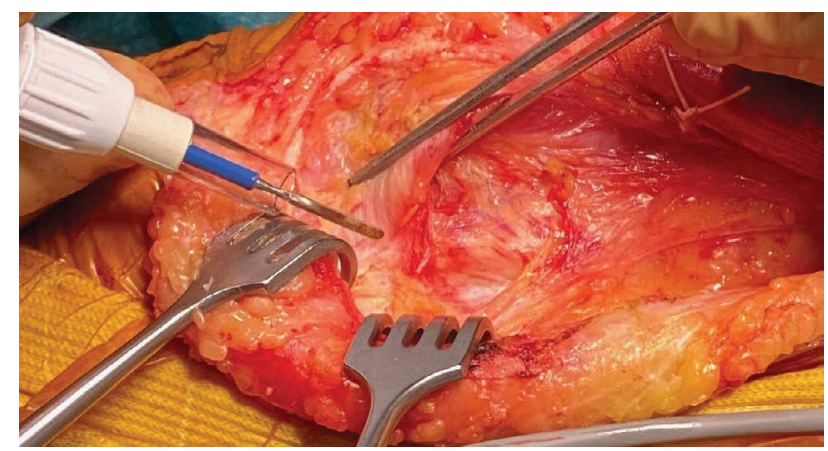

Figure 9: Start arthrotomy.

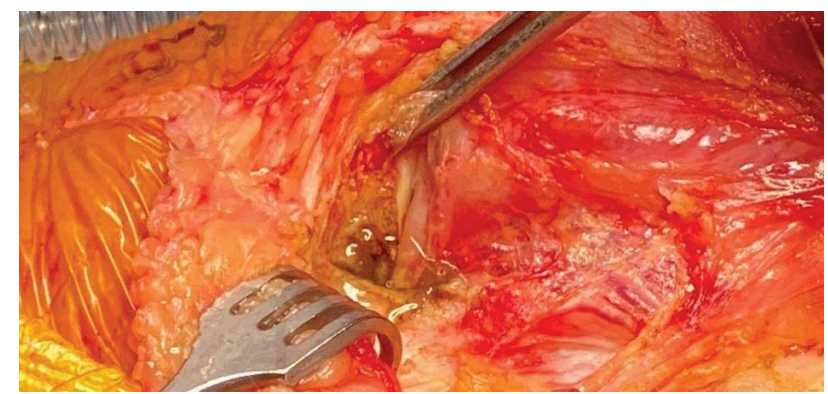

Figure 10: Continue the medial arthrotomy.

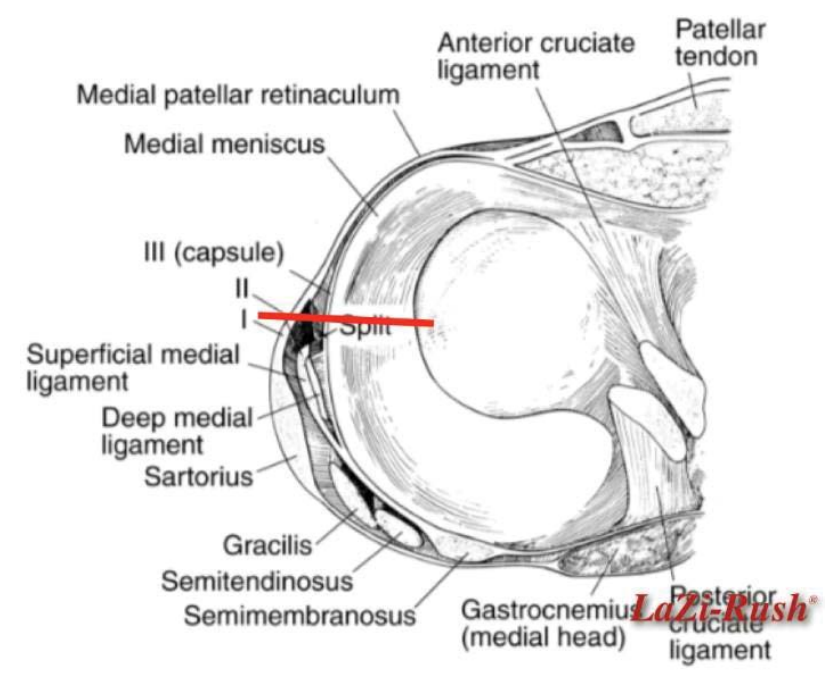

Figure 11: Incise the medial meniscus.

Look now, with the dissection scissors, for three veines on the superior edge of the synovium (anterior of the adductor tuberculum).

These veins have no official anatomic denomination and are appointed by us as the "three friends". The anterior plane of the superior to distal oriented branches of the supero-medial located 3 veins (the so-called "three friends) on the medial femoral condyle can be used as a landmark to the medial arthrotomy which is parallel to the MCL (Figure 8 and Figure 9).

Next the superior and inferior medial genicular arteries are coagulated at the level of the medial meniscus. Open the synovium above the three veins running parallel and distally.

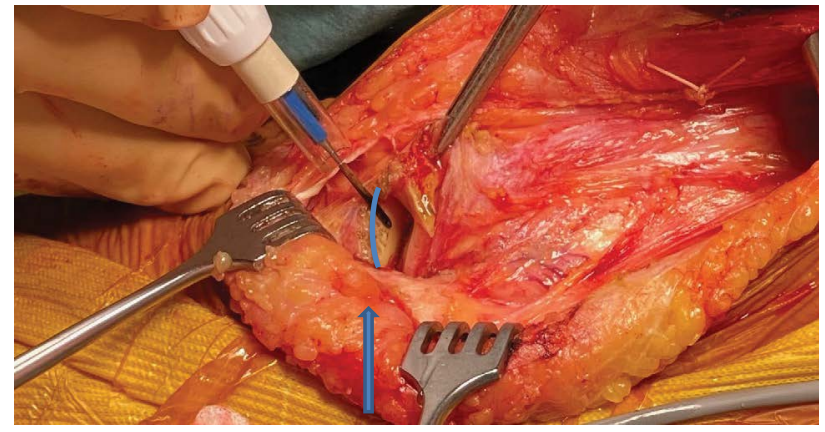

Figure 12: Tibial attachment preserving periosteum.

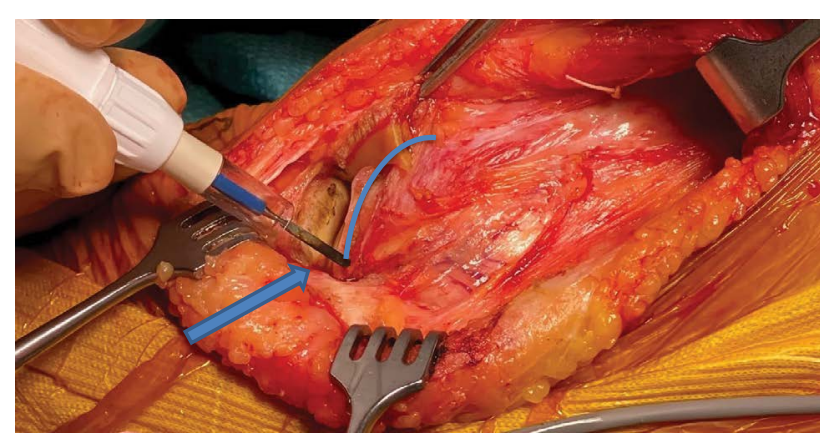

Figure 13: Incision of the femoral attachment of synovium.

Perform now the arthrotomy parallel and $1 \mathrm{~mm}$ anterior to the MCL through the medial meniscus (Figure 10 and Figure 11).

The capsulotomy is then extended towards the patellar tendon, following the inferior border of the meniscus but leaving the anterior tibial periosteum attached to the tibial bone. This might be beneficial to the local blood supply preventing osteonecrosis (Figure 12).

Likewise the synovium is separated from the femoral condyle following the edge of the cartilage (Figure 13).

Next, using a pair of tweezers, the synovial fold is grasped and carefully released with electrocautery without damaging the periosteum (Figure 14).

The suprapatellar pouch and the articularis genus muscle (or subcrureus) are retracted with a Hohman retractor. Articularis genus pulls the suprapatellar bursa superiorly during extension of the knee and prevents impingement of the synovial membrane between the patella and the femur (Figure 15).

Now the synovium on the cartilage edge is released until the lateral part of the trochea is reached. This is the key maneuver for being able to fully mobilize the extensor mechanism.

Next step is to find the attachment of the Medial Patellar Tibial ligament (MPTL) and liberate its tibial insertion to the medial border of the patellar tendon. Release the anterior horn of the medial meniscus using cautery.

A Hohman retractor can now safely be positioned in the deep infrapatellar bursa between the upper part of the tibia and the patellar ligament to retract it together with the In- 
Citation: Ghijselings I, Van den Wyngaert H, Demurie A, et al. (2021) The Eeklo Modified Subvastus Approach for Total Knee Arthroplasty: Detailed Surgical Tips and Tricks. J Orthop Surg Tech 4(1):246-253

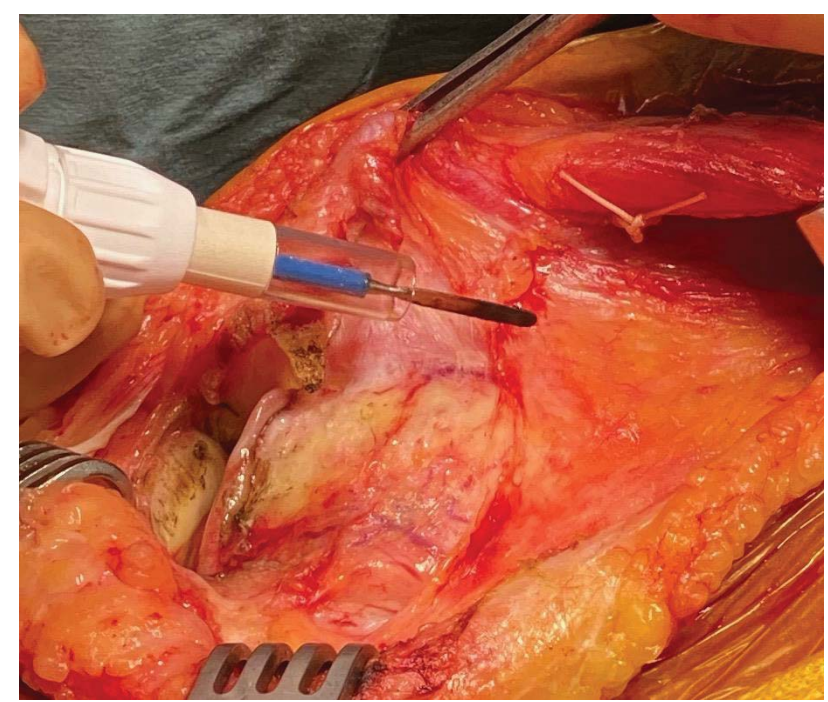

Figure 14: Further release of synovium.

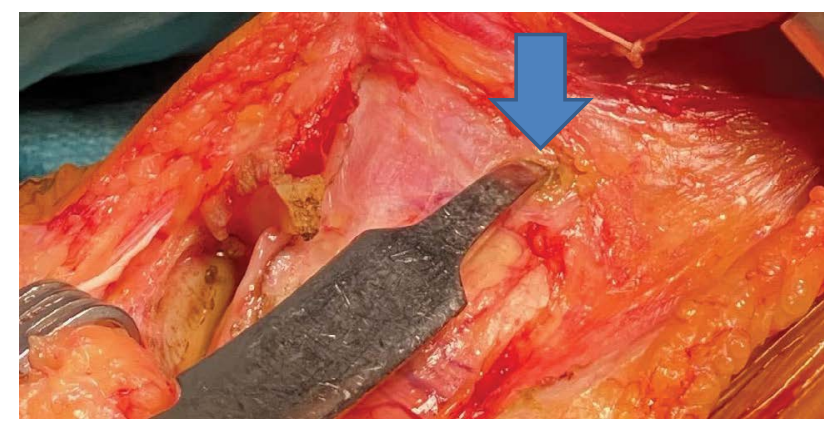

Figure 15: Positioning of the Homan retractor.

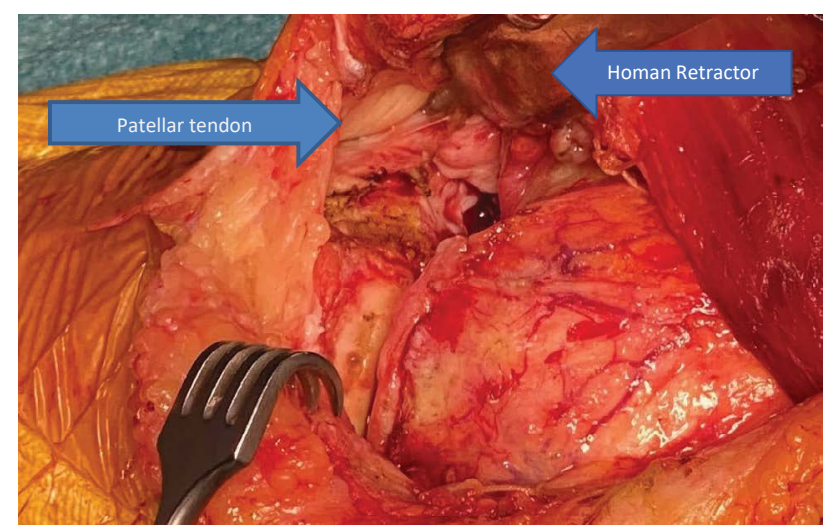

Figure 16: Retracting patellar tendon and IPFP.

frapatellar Fat Pad (I.P.F.P.) and progressively expose the anterolateral tibial margin (Figure 16).

The anterior $1 / 3$ of lateral meniscus is detached from its meniscal rim in the same manner without disturbing the anterior periosteum of the tibia.

The anteromedial connection of periosteum with the insertion of the patellar tendon is released parallel to its medial border. This prevents disinsertion of the tendon from the tibial tubercle.

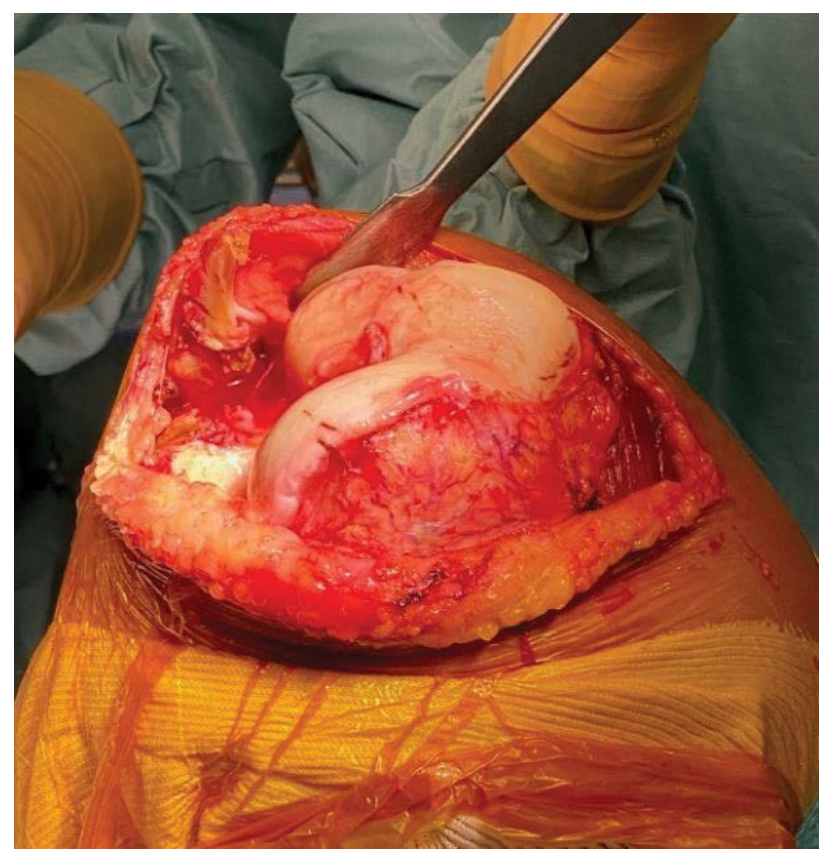

Figure 17: Full exposure of the knee joint.

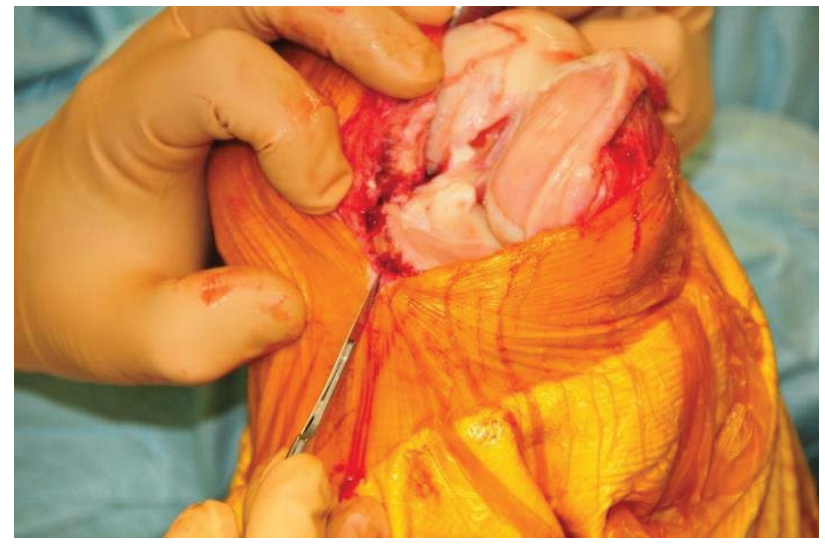

Figure 18: Enlarge incision skin only, not subcutis to avoid nerve damage.

After completing this, the patella can then be subluxated into the lateral gutter.

Reorient the retractor now anterolaterally and flex the knee with the foot in external rotation. Take care not to evert the patella but slide it laterally. Flexing the knee to approximately 90 degrees then provides excellent exposure of the entire joint (Figure 17).

The incision at the distal end is now extended. Only the epiderma and derma are incised but not the underlying subcutis to avoid skin necrosis and damage to the underlying infra patellar nerve (Figure 18 and Figure 19).

The branch of the medial genicular artery in the infrapatellar plica (IPP) or ligamentum mucosum must be coagulated.

Resection of $\mathrm{ACL}$ is performed while preserving PCL. Clear the space anterior to the insertion of the $\mathrm{PCL}$ without damag- 


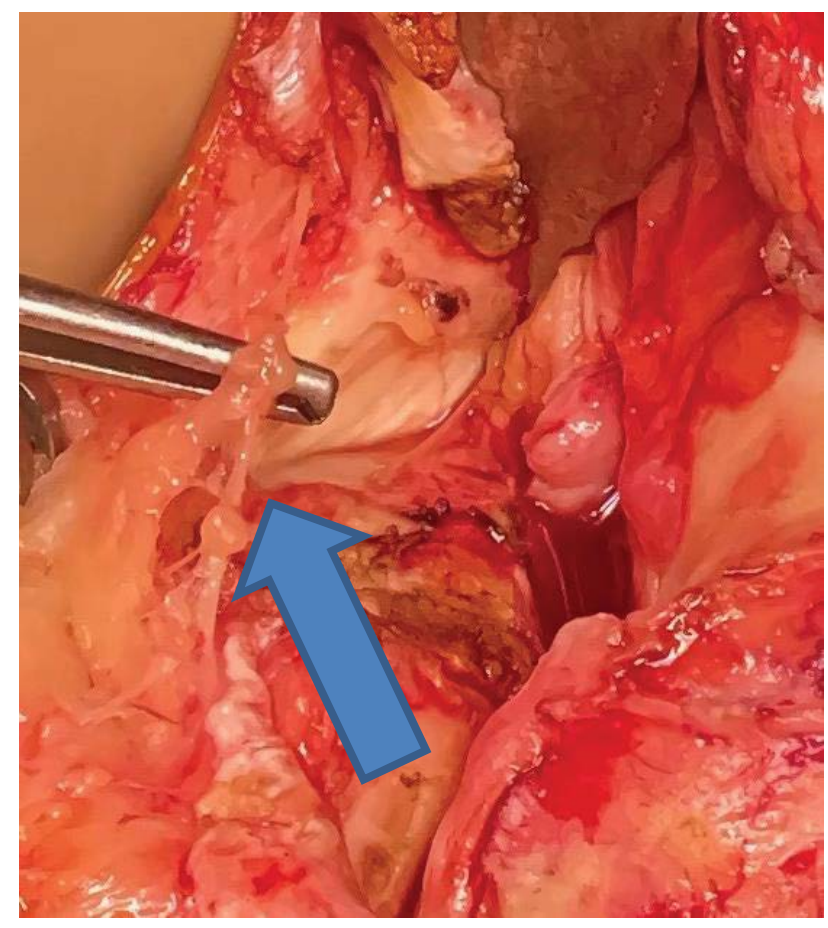

Figure 19: Infrapatellar nerve ending is preserved.

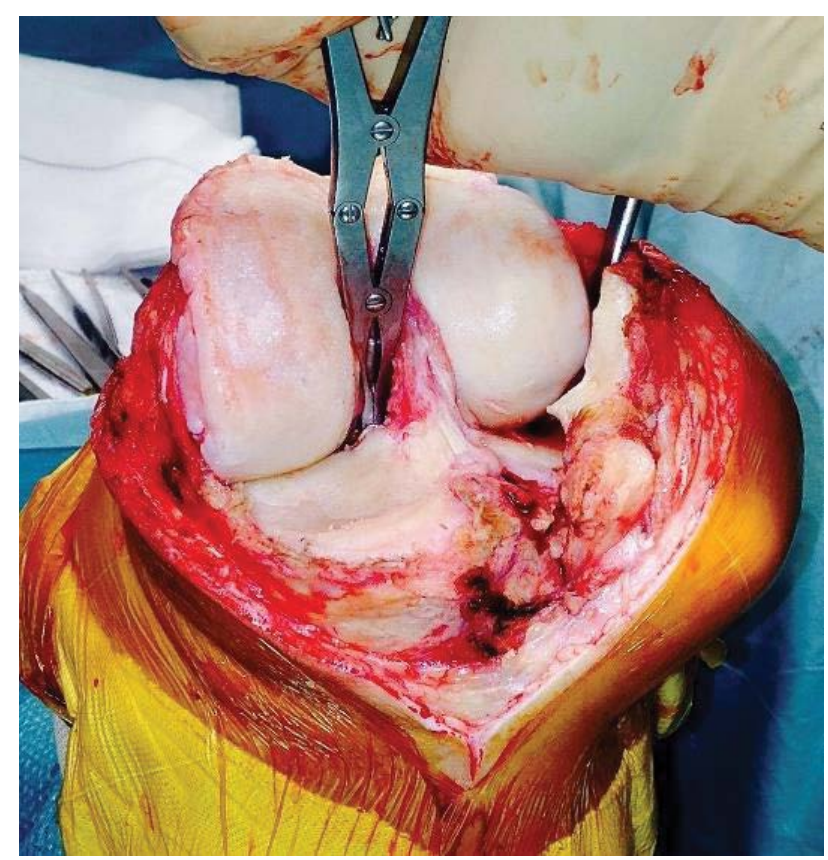

Figure 20: Cleaning the space between $\mathrm{PCL}$ and the posterior horn of the medial meniscus.

ing it (Figure 20).

Rotate the tibia external and incise the now visible posterior horn of the medial meniscus. The lateral meniscus is removed beginning anteriorly, taking care of the Popliteus Tendon and the Lateral Inferior Genicular artery. The resection should remain in the white zone preserving the capsular blood supply and avoiding bleeding. It is appropriate to start to remove the osteophytes already in this stadium.

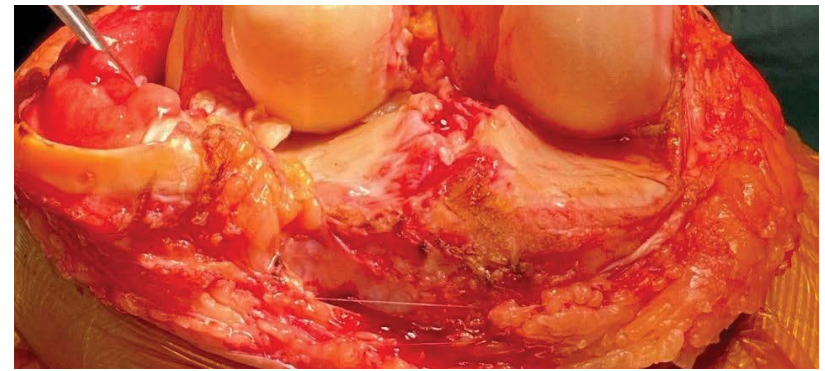

Figure 21: Preserve the periosteum.

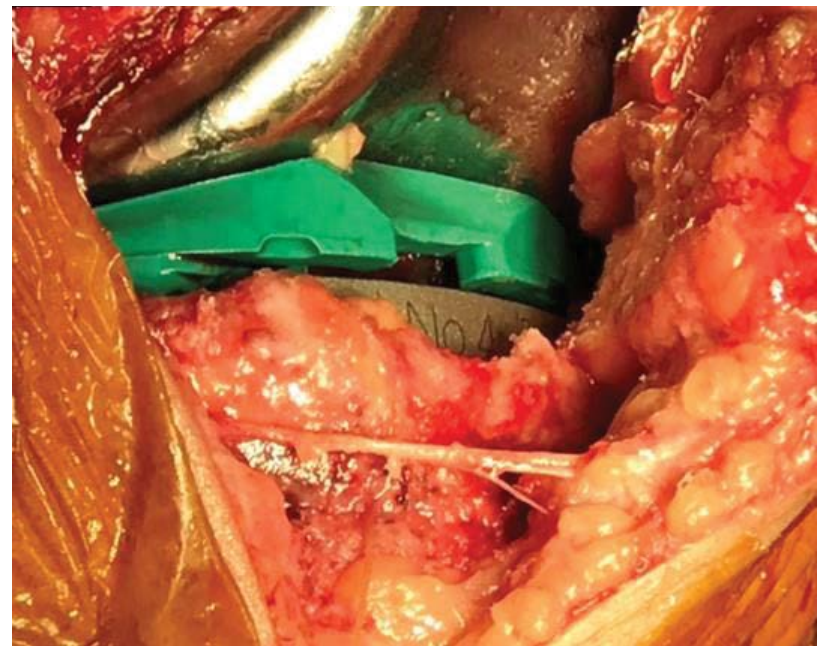

Figure 22: Location of the infrapatellar nerve.

Subsequently, the appropriate procedure as usual, with the knee system utilized by the surgeon, can be performed.

A standard closure is performed the end of the case in hyper-flexion. The closure is quite simple as no muscle needs to be repaired. We close the joint in different layers starting with the synovium. No drain is used. The retinaculum is closed and the MPFL-complex re-attached anatomically.

With the knee in $30^{\circ}$ of flexion the fascia and subcutis are closed. The skin is closed with the knee again in maximal flexion.

\section{Discussion}

The advantages of this approach are multiple:

1) We have found the EMS approach to provide excellent exposure in varus as well in valgus knees:

- The EMS approach preserves the blood supply to the patella. This will diminish risk of fractures, necrosis as well as loosening of the patellar component.

- The periosteal coverage of the proximal tibia is protected to preserve blood supply -obviously avoiding necrosis- accordingly possible loosening of the medial tibial plateau through subclinical local osteonecrosis. Keeping in mind that the periosteum is very sensitive it is likewise important to leave it intact to decrease 
anterior knee pain (Figure 21).

2) It allows faster rehabilitation:

- The extensor mechanism remains intact to conserve the quadriceps strength. Anatomical reconstruction of the MPFQ Complex is crucial [9,13-15].

- As important however is the avoidance of denervation of the VMO by branches of the posterior division of the femoral nerve [16].

3) The medial parapatellar skin incision obviates bony structures with as benefit easier flexion on the occasion of induration of the scar tissue. Painful scar tissue can form post-surgery at the level of the skin or deeper. The EMS incision and arthrotomy avoids all bony prominences preventing limitation of flexion in case of induration of healing cicatrices.

4) Less anterior knee pain after knee surgery:

- Preservation of the infrapatellar nerve avoids neurinoma formation (Figure 22). The infrapatellar branch arises from the fascia lata; level with theinferior pole of the patella and then traverses from medial to lateral, inferior to the patella. Mochida and Kikichu in their examination of 129 cadaver knees found that the infrapatellar brancheither traversed the patella tendon either just below the inferiorpole of the patella (68\%) or just above the tibial tubercle (32\%). Transection of the infrapatellar branches is a significant factor in patient dissatisfaction with total knee arthroplasty $[10,11,17,18]$.

- The retro-patellar bursa and Infra Patellar Fat Pad (I.P.F.P.) are preserved, since they act as a hydraulic shock absorber, filling the anterior compartment $[12,19,20]$. The I.P.F.P. may have a biomechanical function and may play a role in anterior knee pain syndrome. Preservation of the IPFP has been shown to improve TKA-outcome [21].

Absence of the need for a lateral retinacular release has been observed after balancing the knee with the Patella-in-Place Balancer (PIPB). With a specific balancing technique developed in our group there is no need for releases [22].

As with any procedure, there is a definite learning curve associated with this type of Subvastus approach. The author's technique and indications have evolved with time and experience. Notwithstanding the hype of minimal invasive surgery (MIS), we place less emphasis on the length of the skin incision. The senior authors perform this type of Subvastus approach whenever possible; however sometimes (in case limited flexion) an osteotomy of the tibial tubercle is safer.

Obesity, contractures and deformity are relative contraindications, however, with experience, the indications can be expanded. The authors have not encountered problems with muscular nor obese patients. Previous scar tissue on the medial side of the knee due to previous surgerycan make the definition of the different planes more difficult.

In his study, Thienpont demonstrated faster recovery of the quadriceps muscle using a far medial Subvastus approach. Although the technique is not described as detailed in this report, we noticed several differences [23]. The most important finding of this study was that the minimally invasive far medial Subvastus approach allows faster recovery with quick ambulation, fast straight leg raising and stair negotiation resulting in a shorter length of stay even in one day clinic and this without malalignment or complications, after TKA.

The careful dissection of the distal part of the arthrotomy and especially the avoidance of the surgical release of the medial collateral ligament during the approach possibly plays a role in pain, stiffness and recovery. Recently, a new method is developed by our group to re-install isometry of the $\mathrm{MCL}$ very accurately $[2,22]$.

\section{Conclusion}

We have found the Eeklo-ModifiedSubvastus (EMS) approach to provide excellent exposure and allow quicker rehabilitation after total knee surgery. As with any procedure, there is a definite learning curve associated with the EMS approach. The senior author's technique and indications have evolved with time and experience. Less emphasis is placed on the length of the skin incision and more emphasis placed on careful dissection and atraumatic mobilization of the muscle.

\section{Competing Interests}

The authors declare that they have no competing interests.

\section{Author's Contributions}

All authors have made a substantial contribution to this paper and were involved in drafting the manuscript or revising it critically on important intellectual contents. All authors read and approved the final manuscript.

\section{Acknowledgements}

None.

\section{References}

1. Aglietti P, Baldini A, Sensi L (2006) Quadriceps-sparing versus mini-subvastus approach in total knee arthroplasty. Clinical Orthopaedics \& Related Research 452: 106-111.

2. Delport HP, Vander Sloten J, Bellemans J (2013) New possible pathways in improving outcome and patient satisfaction after TKA. Acta Orthop Belg 79: 250-254.

3. Weinhardt C, Barisic M, Bergmann EG, et al. (2004) Early results of subvastus versus medial parapatellar approach in primary total knee arthroplasty. Archives of Orthopaedic \& Trauma Surgery 124: 401-403.

4. Anderson KC, Buehler KC, Markel DC (2005) Computer assisted navigation in total knee arthroplasty: Comparison with Conventional Methods. J Arthropl 132-138.

5. Victor J, Wong P, Witvrouw E, et al. (2009) How isometric are the medial patellofemoral, superficial medial collateral, and lateral collateral ligaments of the knee? Am J Sports Med 37: 20282036.

6. Delport HP, Labey L, De Corte R, et al. (2013) Collateral ligament 
Citation: Ghijselings I, Van den Wyngaert H, Demurie A, et al. (2021) The Eeklo Modified Subvastus Approach for Total Knee Arthroplasty: Detailed Surgical Tips and Tricks. J Orthop Surg Tech 4(1):246-253

strains during knee joint laxity evaluation before and after TKA Clin Biomech (Bristol, Avon) 28: 777-782.

7. Delport HP, Labey L, Innocenti B, et al. (2013) Restoration of constitutional alignment in TKA leads to more physiological strains in the collateral ligaments. Knee Surg Sports Traumatol Arthrosc 23: 2159-2169.

8. Zimny ML, Wink CS (1991) Neuroreceptors in the tissues of the knee joint. Journal of 331 Electromyography and Kinesiology 3: 148-157.

9. Goorens CK, Robijn H, Hendrickx B, et al. (2010) Reconstruction of the medial patellofemoral ligament for patellar instability using an autologous gracilis tendon graft. Acta Orthop Belg 76: 398-402.

10. Kanto N, Muratsu H, Matsumoto T, et al. (2014) Early-onset severe neuromatous pain of the infrapatellar branch of the saphenous nerve after total knee arthroplasty. Asia-Pacific Journal of Sports Medicine, Arthroscopy, Rehabilitation and Technology 1: 102-105.

11. Lee SR, Dahlgren NJP, Staggers JR, et al. (2019) cadaveric study of the infrapatellar branch of the saphenous nerve: Can damage be prevented in total knee arthroplasty?. Journal of Clinical Orthopaedics and Trauma 10: 274-277.

12. Lemon M, Packham I, Narang K, et al. (2007) Patellar tendon length after knee arthroplasty with and without preservation of the infrapatellar fat pad. J Arthroplasty 22: 574-580.

13. Aframian A, Smith TO, Tennent TD, et al. (2017) Origin and insertion of the medial patellofemoral ligament: A systematic review of anatomy. Knee Surg Sports TraumatolArthrosc 25: 3755-3772.

14. Loeb AE, Tanaka MJ (2018) The medial patellofemoral complex. Curr Rev Musculoskelet Med 11: 201-208.
15. Tanaka MJ (2017) The anatomy of the medial patellofemoral complex. Sports Med Arthrosc Rev 25: e8-e11.

16. Thiranagama R (1990) Nerve supply of the human vastus medialis muscle. J Anat 170: 193-198.

17. Dinshaw M, O'Meeghan C (2005) Fate of the infrapatellar branch of the saphenous nerve post total knee arthroplasty. ANZ Journal of Surgery 75: 822-824.

18. Mochida H, Kikuchi S (1995) Injury to infrapatellar branch of saphenous nerve in arthroscopic knee surgery. Clin Orthop 320: 88-94.

19. Bohnsack M, Wilharm A, Hurschler C, et al. (2004) Biomechanical and kinematic influences of a total infrapatellar fat pad resection on the knee. Am J Sports Med 32: 1873-1880.

20. Nisar S, Lamb JN, Somashekar N, et al. (2019) Preservation vs. resection of the infrapatellar fat pad during total knee arthroplasty part II: A systematic review of published evidence. Knee 26: 422-426.

21. Partridge T, Carluke I, Emmerson K, et al. (2016) Improving patient reported outcome measures (PROMs) in total knee replacement by changing implant and preserving the infrapatellafatpad: A quality improvement project. BMJ Quality Improvement Reports 5:

22. Ettinger M, Calliess T, Demurie A, et al. (2015) Patella in place balancer: Technique for total knee arthroplasty. Orthopade 44: 269-274.

23. Thienpont $E$ (2013) Faster quadriceps recovery with the far medial subvastus. Knee Surg Sports Traumatol Arthrosc 21: 23702374. 